\title{
(- OPEN ACCESS \\ Informed consent for functional MRI research on comatose patients following severe brain injury: balancing the social benefits of research against patient autonomy
}

\author{
Tommaso Bruni, ${ }^{01}$ Mackenzie Graham, ${ }^{2}$ Loretta Norton, ${ }^{3,4}$ Teneille Gofton, ${ }^{4}$ \\ Adrian M Owen, ${ }^{3,5}$ Charles Weijer ${ }^{3,5}$
}

${ }^{1}$ Medizinhistorisches Institut, Universitätsklinikum Bonn, Bonn, Germany

${ }^{2}$ Oxford Uehiro Centre for Practical Ethics, University of Oxford, Oxford, UK

${ }^{3}$ Brain and Mind Institute, Western University, London,

Ontario, Canada

${ }^{4}$ Department of Clinical Neurological Sciences, Western University, London, Ontario, Canada

${ }^{5}$ Rotman Institute of Philosophy, Western University, London, Ontario, Canada

Correspondence to Dr Tommaso Bruni, Medizinhistorisches Institut, Universitätsklinikum Bonn, Bonn, 53127, Germany; tombruni@uni-bonn.de

Received 21 March 2018 Revised 1 December 2018 Accepted 12 December 2018 Published Online First 25 February 2019

\section{Check for updates}

(C) Author(s) (or their employer(s)) 2019. Re-use permitted under CC BY-NC. No commercial re-use. See rights and permissions. Published by BMJ.

To cite: Bruni T, Graham M, Norton $\mathrm{L}$, et al. J Med Ethics 2019;45:299-303.

\begin{abstract}
Functional MRI shows promise as a candidate prognostication method in acutely comatose patients following severe brain injury. However, further research is needed before this technique becomes appropriate for clinical practice. Drawing on a clinical case, we investigate the process of obtaining informed consent for this kind of research and identify four ethical issues. After describing each issue, we propose potential solutions which would make a patient's participation in research compatible with her rights and interests. First, we defend the need for traditional proxy consent against two alternative approaches. Second, we examine the impact of the intensive care unit environment on the informed consent process. Third, we discuss the therapeutic misconception and its potential influence on informed consent. Finally, we deal with issues of timing in recruiting participants and related factors which may affect the risks of participation.
\end{abstract}

\section{CASE}

Kathy (this is a representative composite case that does not reflect any single patient) was a 67-year-old woman who suffered an out-of-hospital cardiac arrest. She received 5 min of cardiopulmonary resuscitation and defibrillation by emergency medical services at the scene before the return of spontaneous circulation. In the hospital, she was diagnosed with an ST-elevation myocardial infarction and underwent thrombolysis, followed by 24 hours of induced therapeutic hypothermia, a treatment intended to reduce the risk of brain injury after cardiac arrest. Unfortunately, Kathy sustained severe anoxic brain injury and remained comatose after restoration of normal body temperature.

Additional tests were done to help determine her neurological prognosis. These tests showed mixed results. On the one hand, an MRI scan showed no structural abnormality, and somatosensory evoked potentials were bilaterally present with normal latency and amplitude. While the bilateral absence of the $\mathrm{N}_{20}$ somatosensory evoked potentials response indicates a poor prognosis, ${ }^{1}$ its presence does not predict neurological outcome. On the other hand, Kathy displayed persistent seizure activity on her electroencephalogram, which is associated with increased mortality following cardiac arrest. ${ }^{23}$

The neurocritical care team met with Kathy's husband and son and explained that they were uncertain about her likely outcome and needed more time and information to develop a clearer picture of Kathy's prognosis. The care team was aware of an ongoing research study at the hospital involving functional MRI for which Kathy was eligible. The study involved recording brain responses to stimuli and comparing patient responses with eventual outcome to determine if functional MRI could be a useful prognostic test for future patients. Functional MRI shows promise as a candidate prognostication method in patients with acute brain injury. ${ }^{4-6}$

There are ethical issues associated with enrolling patients like Kathy in functional MRI studies in the intensive care unit. In particular, given that Kathy herself cannot provide informed consent, how should patient autonomy be protected and consent be obtained? Are there potential obstacles to proxy decision making in this context? Our ethical analysis stems from reflection on a research study that is currently under way at the Lawson Health Research Institute. Written informed consent was obtained from proxy decision makers in all cases. In this article, we identify four ethical issues associated with obtaining informed consent in Kathy's case. After describing each issue, we propose potential solutions which would make Kathy's participation in the functional MRI study compatible with her rights and interests.

\section{STRATEGIES FOR INFORMED CONSENT IN THE INTENSIVE CARE UNIT \\ The problem}

Informed consent is an important ethical protection barring involuntary participation in clinical research, but comatose patients like Kathy are unconscious, mechanically ventilated and often medically unstable. Consequently, they cannot provide informed consent to participate in clinical research. When a patient is incapable of providing informed consent, a proxy decision maker is appointed to act on the patient's behalf and assumes the authority to act as a substitute for the patient with respect to decision making. In many jurisdictions, the law indicates who is the proxy decision maker for an incapable patient (e.g., Ontario Health Care Consent Act, 1996, \$20), and proxy decision makers are typically spouses or other close family members.

The practice of proxy decision making has received criticism, particularly in the context of research in the intensive care unit. ${ }^{78}$ For example, 
Burns and colleagues argue that traditional proxy consent impedes the recruitment of participants and thus slows scientific progress. In the interest of expediting patient enrolment in research, Burns and colleagues suggest two alternatives to obtaining proxy consent:

1. The consent requirement is waived altogether.

2. An emergency exception to the informed consent requirement is sought.

\section{Proposed solutions}

We argue that the two alternatives put forth by Burns and colleagues do not sufficiently protect patient autonomy in this case and are therefore not suitable for functional MRI research in the intensive care unit. Traditional proxy consent is the only ethically appropriate strategy in this case. Consider the first proposed alternative: waiving consent completely. According to Canadian regulation, a waiver of consent is not acceptable in studies involving more than minimal risk (Tri-Council Policy Statement Article 3.7A). Minimal risk is defined as the risks of daily life and is widely understood to subsume the risks of a blood draw, routine physician examination and filling out a questionnaire, all benign procedures. This provision is based, in our view correctly, on the ethical consideration that it would be immoral to expose a human being to more than minimal risk without her consent because doing so would wrongly disregard the respect that is owed to her autonomy.

In order to evaluate the present case, we must consider that functional MRI research on acute comatose patients poses greater than minimal risks in that it requires transport to the MRI scanner room as well as spending time in the MRI scanner. Research suggests that intrahospital transport of patients from the intensive care unit results in a serious adverse event in $4.2 \%$ $8.9 \%$ of cases and cardiac arrest in $0.34 \%-1.6 \%$ of cases. ${ }^{9}$ Time spent in the scanner is a risk because the patient is outside the relative safety of the intensive care unit. If the patient experiences a medical event (eg, a drop in blood pressure or an increase in intracranial pressure) outside the intensive care unit, the potential for a negative outcome is increased. If study participation exposes patients to more than minimal risk, a waiver of consent is not ethically acceptable. Even if study participation did not expose patients to more than minimal risk, a waiver of consent could be granted only if a prior consent requirement made it impossible or extremely difficult to carry out the study. In the absence of strong feasibility considerations, there would be no cogent ethical reason to deprive the patient of the informed consent protection. Indeed, Tri-Council Policy Statement Article 3.7A grants these waivers, in our view correctly, only if prior consent makes the study impossible or impracticable. But this condition is not satisfied for proxy consent in functional MRI research, as it is practicable to run the study after valid proxy consent has been obtained. As a result, a waiver of consent would not be morally acceptable even if the participants were exposed to risk not exceeding the minimal risk standard.

Consider the second proposed alternative: granting an emergency exception to consent. The purpose of an emergency exception to consent is to allow research in situations in which a potential research participant requires urgent medical care and is unable to consent for herself, and the delay involved in locating a proxy decision maker could pose a serious risk to the participant's health. Absent an immediate danger to the prospective participant or when the research intervention has no therapeutic effects, an emergency exception does not apply because it would needlessly remove the informed consent protection to which participants are normally entitled. Indeed, Tri-Council Policy
Statement Article 3.8 rightly specifies that an emergency exception can be granted only if 'a serious threat to the prospective participant requires immediate intervention' and the research 'addresses the emergency needs of the individuals involved'. ${ }^{10}$

Although patients like Kathy are in critical condition and are often medically unstable, functional MRI does not constitute a response to an urgent medical need and is not an instance of emergency research. The study does not include any life-saving interventions, and the delay associated with locating a proxy decision maker does not pose a health risk to patients. In fact, functional MRI in the proposed research is a non-therapeutic research intervention; it does not offer a realistic probability of direct benefit to the participant. ${ }^{11}$ The benefits of the study lie in the production of generalisable knowledge, which may help future patients, but not Kathy herself. As the conditions for an emergency exception do not obtain, functional MRI research on acute comatose patients must rely on traditional proxy consent.

\section{OBSTACLES TO PROXY DECISION MAKING IN THE INTENSIVE CARE UNIT CONTEXT \\ The problem}

The intensive care unit is a challenging environment for clinical research and creates obstacles to obtaining informed consent from proxy decision makers. Complicating factors, including stress $^{12}$ and misunderstandings about the purpose of the research, ${ }^{13}$ can impede the decision-making process for proxy decision makers and thereby weaken informed consent protections. For example, Iverson and colleagues studied family members of patients currently in the intensive care unit and used a focus group to discuss their perspectives on participation in clinical research. ${ }^{14}$ The authors found that family members perceived their experience in the hospital as stressful and overwhelming. Specifically, anxiety and stress were mentioned as reasons not to enrol patients in proposed research studies. Three main sources of stress were identified:

1. Observing the patient in a critically ill state.

2. Uncertainty about the patient's clinical outcome.

3. Contemplating whether the patient's views were being accurately reflected by the proxy decision maker.

\section{Proposed solutions}

Given these potential obstacles to proxy decision making, what strategies might researchers employ to enable proxy consent in the intensive care unit and ensure that patient autonomy is respected? One way to promote proxy consent is to approach a patient's family about participation in a research study at a time when stress is minimal, and they are mentally and emotionally prepared to consider this option. The insights of the patient's nurse as well as her intensive care unit physicians are particularly useful, as they will have had the most contact with the patient's family. Thus, the patient's immediate healthcare providers are in a good position to inform judgments about whether and when families should be approached for research participation. If a proxy decision maker is not in a condition to discuss participation in research, she should not be contacted by the research team at that time. In some cases, a social worker may provide useful additional insights.

In addition to deciding when to approach the patient's family, it is also important to consider who will approach them. When the treating physician is also the researcher, an approach by the physician may create the impression of conflict of interest and even undermine trust between the physician and the family. Worse, families may feel pressured to enrol their family member, 
or may believe that the physician merely wants to use her as a means to achieving a research goal. To avoid these potential pitfalls, we argue that the idea of research participation ought to be first discussed with the patient's healthcare providers. If there is agreement that the family is in a sufficiently robust emotional state, the family ought to be approached by a third party, in our case a highly trained research coordinator. The research coordinator will explain the study to the family and will seek their proxy consent for participation. In this conversation, the research coordinator will explain the study's purpose, procedures, benefits (if any), risks, alternatives, the right to refuse participation and to whom questions may be addressed. Adequate time should be set aside for this interaction, so the family does not feel rushed. Using a research coordinator maintains a clear distinction between the clinical care of the patient and participation in research, thereby making it easier for the family to protect patient autonomy in this challenging environment.

\section{THE THERAPEUTIC MISCONCEPTION The problem}

The risk of 'therapeutic misconception' poses a further obstacle to the exercise of informed consent rights in functional MRI research in the intensive care unit. ${ }^{15}$ Appelbaum and colleagues define the therapeutic misconception as the belief that 'every aspect of the research project to which [the patient] had consented was designed to benefit him directly' (p20). ${ }^{15}$ Under a therapeutic misconception, patients and proxy decision makers fail to understand that participation in a research study is different from routine medical care.

There is some debate regarding the degree to which therapeutic misconception undermines the validity of informed consent and weakens patient autonomy. It has been argued that because consent under therapeutic misconception is uninformed, it is not valid; the person consenting lacks understanding and, as such, cannot provide valid consent. ${ }^{16}$ Conversely, others have argued that a patient or proxy decision maker may retain sufficient autonomy to give informed consent even when therapeutic misconception leads to incomplete understanding, and so we should continue to presume autonomy, absent compelling evidence of serious misunderstanding. ${ }^{17}$ Legitimate ethical reasons back both of these standpoints: the former prioritises patient protection, whereas the latter endeavours to strike a balance between respect for patient autonomy and the beneficial social effects of clinical research.

In our view, the key issue is not, however, whether therapeutic misconception makes consent invalid, but rather whether it causes participants to misconstrue the benefits and risks of research participation. If a patient (mistakenly) thinks that she stands to benefit directly from what is actually a non-therapeutic procedure, this may cause her to make a decision that she would not have made if she had understood the facts correctly. In the case at hand, Kathy's family could interpret the non-therapeutic functional MRI scan as a therapeutic procedure which may provide a direct benefit to her, or as a diagnostic procedure that is required by Kathy's medical condition. Both beliefs would instantiate the therapeutic misconception and could lead Kathy's family to accept a higher level of risk than they might otherwise.

There is no empirical evidence regarding the degree or nature of therapeutic misconception in studies using functional MRI on comatose patients, and we believe further study is indicated. There are indirect lines of evidence that suggest that some family members will have unreasonable expectations about the effects of the study on the patient's clinical care. First, the public has faith in technology_particularly sophisticated technology like functional MRI-and expects to benefit from contact with it. Neuroscientific explanations are common and are viewed as compelling, ${ }^{18}$ and functional MRI images are often rated as particularly credible pieces of evidence. ${ }^{19}$ This optimism could prevent Kathy's family from understanding the limitations of the technique and bias them towards a 'therapeutic interpretation' of the functional MRI study. Second, the media, universities and research funding agencies have generated hype about functional MRI. ${ }^{20}$ This hype reinforces the perception of functional MRI mentioned above and may lead to an overestimation of the potential impact of such technologies on patient care. ${ }^{21}$

\section{Proposed solutions}

Two strategies are available to address therapeutic misconception. First, researchers should ensure that consent materials clearly and accurately describe functional MRI as either a therapeutic procedure or a non-therapeutic procedure. This will depend on the specifics of the study protocol. If there is a reasonable prospect of clinical benefit to the patient, it should be described in cautious, evidence-based language. ${ }^{11}$

Second, research staff should spend more time with family members discussing study participation. One-on-one conversations are an effective means of improving the understanding of consent materials and the retention of information by research participants. ${ }^{22} 23$ Additionally, there is some evidence that enhanced consent forms-consent forms that are shorter and written using simpler language than standard forms-are a useful means of improving participant understanding. ${ }^{223}$ In fact, Nishimura and colleagues found that both multimedia interventions and enhanced forms improved information retention over time. ${ }^{23}$ Given these results, the use of enhanced consent forms could also be considered.

\section{TIMING AND DIFFERENTIAL LEVELS OF RISK The problem}

Consent for functional MRI research must be obtained from patients in the intensive care unit in a timely manner. The ideal candidate for these studies is a patient with acute brain injury that is comatose, unsedated and capable of remaining still in the MRI scanner. Finding participants that satisfy these conditions is difficult because the likelihood that a patient will satisfy them decreases over time. Moreover, MRI scanner time is limited, as both clinical and research demand are high.

As discussed above, research involving functional MRI amongst patients with acute brain injury requires intrahospital transport, and the accompanying risk to patients resulting from this transport exceeds minimal risk. ${ }^{9}$ Accordingly, it is preferable to perform the research functional MRI scan at the same time as a clinically indicated structural MRI scan.

Pairing a research scan with a clinically indicated scan reduces the overall risk to the patient. First, it reduces the number of total transports and thus the total risk to which patients are exposed. Second, it makes the transport part of a clinically indicated procedure, meaning the transport-related risk is no longer a research risk, but a clinical risk. As mentioned above, time spent in the scanner is also a source of risk. In the case of paired scans, only the duration of the research scan would count as a research risk; the time spent in the scanner for the purposes of a clinical scan would be a clinical risk. In these cases, the incremental risk posed to patients in functional MRI research may drop below the minimal risk standard. 
In spite of the advantages of pairing MRI scans, doing so further exacerbates time management problems because it requires two slots in a row (which are not always available), and thus can be arranged only if tight time constraints are respected. These time constraints can hinder the family's decision-making process and further complicate the obtainment of informed consent in the intensive care unit setting. For example, the research team could learn that a clinically indicated scan has been ordered with only a short time to talk to the patient's family about the study. In this case, informed consent might be rushed, and proxy decision makers may not have enough time to fully understand the information provided. In fact, Barrett and colleagues showed that proxy decision makers were less comfortable with being involved in clinical research decisions if the enrolment window was short, ${ }^{24}$ while Burns and colleagues showed that expired time windows were one of the main reasons why opportunities to recruit patients in the intensive care unit setting were missed. ${ }^{8}$

The potentially brief amount of time a proxy decision maker has to deliberate can also impact the level of risk associated with participation in these studies; proxy decision makers that consent at different points in time may be consenting to different levels of risk (all other factors, such as the patient's haemodynamic stability, being equal). For example, if a proxy decision maker consents soon enough to make 'pairing' possible, the associated research risk is simply the additional time spent in the scanner. In this case, transport to and from the scanner is a clinical risk and not a research risk. Conversely, if the proxy decision maker consents when it is no longer possible to pair the research scan with a clinical scan, an additional transport to and from the scanner is required, thereby increasing the research-related risk of participation. Accordingly, the proxy decision maker has a rational incentive to make a decision about participation early. However, this incentive could compel proxy decision makers to give consent to participation before they have given it due consideration.

\section{Proposed solutions}

MRI scanner time is a scarce resource required by both clinicians and researchers. Since paired scans reduce the research risk to which patients are exposed, they ought to be conducted whenever possible. If research staff approached families regarding research participation without undue delay, 'paired' scans would not result in a slower pace of research or in a suboptimal decision-making process for family members. Time constraints could be partially eased by increasing the availability of research staff. Another strategy would be to increase the amount of MRI slots that are available to researchers relative to clinical scans.

If 'paired' scans and independent scans are both conducted in a given study, it is critical that the difference in risk between the two kinds of scans be clearly explained to proxy decision makers. The challenge for the research team here is not to rush the proxy decision maker while conveying the relevant information. Furthermore, two consent forms (one for the 'paired' scan and one for the independent scan) with different sections on risk are required. If the functional MRI scan is not paired, the consent document should include a description of the risks associated with transport to the scanner room. If the structural and functional MRI scans are paired, the research consent document may omit reference to the risks of transportation. In this way, proxy decision makers can correctly understand the research risk the patient would be exposed to.

Finally, telephone consent may be a useful tool in addressing time constraints. A member of the research team could contact the proxy decision maker by telephone and verbally convey the relevant information regarding study participation, including the study purpose, procedures, benefits, harms and alternatives. The Research Ethics Board of the Lawson Health Research Institute currently authorises this practice in intensive care settings in which the proxy decision maker is not physically present and study procedures are subject to strict time constraints. The full letter of information must be presented through an approved telephone script. Written consent must be obtained within 24 hours of the telephone consent. If the proxy decision maker agrees to participation by phone but does not sign the informed consent form within 24 hours, the patient is withdrawn from the study. Telephone consent poses challenges to researchers: they must ensure that information is adequately understood and that respect for patient autonomy is not compromised.

\section{CONCLUSION}

Functional MRI research pits respect for patient autonomy against the social benefits of clinical research. We make recommendations that balance these important goods. As the patient is unconscious in our research, the proxy decision maker is tasked with protecting patient autonomy: she must choose-while taking the patient's values and interests into account-whether the patient will be enrolled in the study. First, we argued that the proxy decision maker cannot be dispensed with, because both a waiver of consent and an emergency exception would not be ethically sound in this case. Second, we examined three potential problems for proxy decision makers: they must make an important choice in the challenging environment of the intensive care unit, could fall prey to therapeutic misconception, and face complicated issues of timing. To address these issues, we proposed solutions that, we contend, allow functional MRI research on comatose patients without impinging on any morally significant interests.

\section{BACK TO THE CASE}

The care team brought up the study during one of their meetings with Kathy's family and explained its rationale and procedures. A researcher was introduced to the family, and after an in-depth discussion of the study Kathy's proxy decision maker gave written informed consent. A clinically indicated structural MRI and a functional MRI were performed in a paired manner 11 days post-injury. The functional MRI showed that Kathy had preserved functionality in networks associated with sound perception, speech perception and language comprehension. ${ }^{25}$ However, Kathy could not perform mental imagery tasks, that is, volitional tasks in which the patient imagines carrying out a motor action such as playing tennis. ${ }^{25}$ At the time of imaging, Kathy remained comatose and dependent on a mechanical ventilator. Neurological examination indicated that she could withdraw her lower extremities from painful peripheral stimuli and spontaneously open her eyes, but that she could not fixate, track, blink or close her eyes on command. Three days after the functional MRI (day 14), Kathy's neurological status started to gradually improve. A month after her injury, her family reported that her usual personality had returned. Kathy was discharged 40 days after admission fully alert, oriented and asking appropriate questions. At the 6-month follow-up, she had resumed her normal activities prior to admission.

Contributor TB and CW conceptualized the paper. TB wrote the first draft of the paper, and MG and CW edited and rewrote sections of the next draft of the paper. All other team members provided comments on subsequent drafts of the paper. All authors approved the final paper. 
Funding This study was funded by the Canadian Institutes of Health Research (grant numbers: 201110GSD-277640-183782 and MOP133705).

Competing interests TG reports grants from the Lawson Health Research Institute, the Academic Medical Organization of Southwestern Ontario and St Joseph's Health Care Foundation; she receives other funding from Medtronic for being an examining neurologist in the SURTAVI trial and from SAGE Therapeutics for being a site principal investigator in a clinical trial. CW receives consulting income from Eli Lilly and Company Canada. All other authors report no competing interests.

Patient consent Not required.

Ethics approval Western University's Health Sciences Research Ethics Board approved the protocol of the functional MRI study.

Provenance and peer review Not commissioned; externally peer reviewed.

Data sharing statement This article is argument-based, so that there are no unpublished data to be shared.

Open access This is an open access article distributed in accordance with the Creative Commons Attribution Non Commercial (CC BY-NC 4.0) license, which permits others to distribute, remix, adapt, build upon this work non-commercially, and license their derivative works on different terms, provided the original work is properly cited, appropriate credit is given, any changes made indicated, and the use is non-commercial. See: http://creativecommons.org/licenses/by-nc/4.0/.

\section{REFERENCES}

1 Wijdicks EF, Hijdra A, Young GB, et al. Practice parameter: prediction of outcome in comatose survivors after cardiopulmonary resuscitation (an evidence-based review): report of the Quality Standards Subcommittee of the American Academy of Neurology. Neurology 2006;67:203-10.

2 Rossetti AO, Logroscino G, Liaudet L, et al. Status epilepticus: an independent outcome predictor after cerebral anoxia. Neurology 2007;69:255-60.

3 Rossetti AO, Oddo M, Liaudet L, et al. Predictors of awakening from postanoxic status epilepticus after therapeutic hypothermia. Neurology 2009;72:744-9.

4 Gofton TE, Chouinard PA, Young GB, et al. Functional MRI study of the primary somatosensory cortex in comatose survivors of cardiac arrest. Exp Neurol 2009;217:320-7.

5 Norton L, Hutchison RM, Young GB, et al. Disruptions of functional connectivity in the default mode network of comatose patients. Neurology 2012;78:175-81.

6 Koenig MA, Holt JL, Ernst T, et al. MRI default mode network connectivity is associated with functional outcome after cardiopulmonary arrest. Neurocrit Care 2014;20:348-57.
7 Burns KE, Magyarody NM, Duffett M, et al. Attitudes of the general public toward alternative consent models. Am J Crit Care 2011;20:75-83.

8 Burns KE, Zubrinich C, Tan W, et al. Research recruitment practices and critically ill patients. A multicenter, cross-sectional study (the Consent Study). Am J Respir Crit Care Med 2013;187:1212-8.

9 Fanara B, Manzon C, Barbot 0, et al. Recommendations for the intra-hospital transport of critically ill patients. Crit Care 2010;14:R87.

10 Canadian Institutes of Health Research, Natural Sciences and Engineering Research Council of Canada, and Social Sciences and Humanities Research Council of Canada. Tri-Council policy statement: ethical conduct of research involving humans. 2014.

11 Weijer C, Bruni T, Gofton T, et al. Ethical considerations in functional magnetic resonance imaging research in acutely comatose patients. Brain 2016;139:292-9.

12 Larkin ME, Beauharnais CC, Magyar K, et al. Obtaining surrogate consent for a minimal-risk research study in the intensive care unit setting. Clin Tria/s 2013;10:93-6.

13 Silverman $\mathrm{H}$. Protecting vulnerable research subjects in critical care trials: enhancing the informed consent process and recommendations for safeguards. Ann Intensive Care 2011;1:8.

14 Iverson E, Celious A, Kennedy CR, et al. Real-time perspectives of surrogate decisionmakers regarding critical illness research. Chest 2012;142:1433-9.

15 Appelbaum PS, Roth LH, Lidz CW, et al. False hopes and best data: consent to research and the therapeutic misconception. Hastings Cent Rep 1987;17:20-4.

16 Dresser R. The ubiquity and utility of the therapeutic misconception. Soc Philos Policy 2002;19:271-94.

17 McConville P. Presuming patient autonomy in the face of therapeutic misconception. Bioethics 2017:31:711-5

18 Weisberg DS, Keil FC, Goodstein J, et al. The seductive allure of neuroscience explanations. J Cogn Neurosci 2008;20:470-7.

19 McCabe DP, Castel AD. Seeing is believing: the effect of brain images on judgments of scientific reasoning. Cognition 2008;107:343-52.

20 Racine E, Bar-llan O, Illes J. fMRI in the public eye. Nat Rev Neurosci 2005;6:159-64.

21 Samuel G, Kitzinger J. Reporting consciousness in coma: Media framing of neuroscientific research, hope, and the response of families with relatives in vegetative and minimally conscious states. JOMEC Journal 2015;3:1-15.

22 Flory J, Emanuel E. Interventions to improve research participants' understanding in informed consent for research: a systematic review. JAMA 2004;292:1593-601.

23 Nishimura A, Carey J, Erwin PJ, et al. Improving understanding in the research informed consent process: a systematic review of 54 interventions tested in randomized control trials. BMC Med Ethics 2013;14:28.

24 Barrett KA, Ferguson ND, Athaide V, et al. Surrogate decision makers' attitudes towards research decision making for critically ill patients. Intensive Care Med 2012:38:1616-23.

25 Owen AM, Coleman MR. Functional neuroimaging of the vegetative state. Nat Rev Neurosci 2008;9:235-43. 